\title{
USO Y ABUSO DE LAS TIC Y REPETICIÓN DE CURSO EN ADOLESCENTES
}

\author{
Ángela Barrio Fernández \\ IES. Santa Clara. Santander
}

Fecha de Recepción: 12 Marzo 2018

Fecha de Admisión: 10 Abril 2018

\section{RESUMEN}

Las nuevas tecnologías son utilizadas cada vez de manera más frecuente por los adolescentes. Los dispositivos y herramientas que facilitan este uso de estas TIC están muy generalizados entre ellos. Los padres, los educadores, y la sociedad, debemos de realizar un mayor esfuerzo en la educación con respecto al consumo de los diferentes medios tecnológicos, incentivando una utilización racional de los mismos, y fomentando el espíritu crítico en los adolescentes que les permitan decidir cómo quieren usar las TIC de un modo responsable.

En los últimos años, la variedad de posibilidades que se encuentra un adolescente para hacer en su tiempo libre ha aumentado considerablemente. Esto está incidiendo en nuevos y diferentes estilos de vida y también de aprendizaje y de estudio y puede repercutir sobre el rendimiento académico.

Se ha realizado un estudio en la C.A. de Cantabria, con una muestra de 2371 alumnos, del rango de cursos desde $1^{0}$ de ESO hasta $1^{\circ}$ de Bachillerato con una distribución de 1168 mujeres y1203 varones, y un muestreo por estratos y conglomerados para que la muestra pudiera ser considerada representativa de la Comunidad Autónoma, y una de las cuestiones analizadas es la de las repeticiones de curso y su posible relación con el uso y abuso de las TIC.

Un $22,77 \%$ de los alumnos de la muestra ha repetido curso, lo que sin duda requiere una reflexión. En aquellos que han repetido curso, se ha podido apreciar que usan más las nuevas tecnologías y de manera menos adecuada, en comparación con los que no han repetido curso. En este artículo, se ha querido poner de manifiesto la influencia del inadecuado uso de las diferentes tecnologías sobre la repetición de cursos escolares de los alumnos, a través del análisis de las diferentes variables que se analizan.

Palabras clave: adolescentes, uso de las TIC, repetición de curso, calificaciones

\section{ABSTRACT}

Use and abuse of ict and repetition of course in adolescents.

New technologies are used more and more frequently by adolescents. The devices and tools that facilitate the use of ICTs are very widespread among adolescents. As parents, educators, and as 


\section{USO Y ABUSO DE LAS TIC Y REPETICIÓN DE CURSO EN ADOLESCENTES}

society as a whole, we must make a greater education effort regarding the consumption of different technological means, encouraging a rational use of them, and encouraging the critical spirit in adolescents that allows them to decide how they want to use ICTs in a responsible way.

In recent years, the variety of possibilities in which a teenager can spend his free time has increased considerably. This is influencing new and different lifestyles, and can also affect study and academic performance.

A study has been conducted in the Autonomous Community of Cantabria, with a sample of 2371 students, ranging from the 1st ESO course to the 1st Bachiller's course, and a distribution of 1168 women and 1203 men. Stratified sampling was performed per conglomerate such that the sample could be considered representative of the Autonomous Community. One of the issues analyzed is course repetition and its possible relationship with the use and abuse of ICTs.

$22.77 \%$ of the students in the sample have repeated a course, which undoubtedly requires consideration. It was observed that those who have repeated a course use more new technologies and they use them less adequately, compared to those who have not repeated any course. In this article, we wanted to highlight the influence of the inappropriate use of different technologies on the repetition of students' school courses, through the analysis of the different variables analyzed.

Keywords: adolescents, use of ICTs, course repetition, grades

\section{INTRODUCCIÓN}

Estamos gozando del privilegio de vivir unos momentos históricos en la evolución de la humanidad. Es el inicio de una nueva etapa. La "sociedad red» se identifica con acelerados cambios que se suceden entre el mundo real y en el virtual. El progreso de dispositivos digitales ha generado un nuevo modelo de ocio (Valdemoros, M.A., et al., 2017), y también de cultura y vida.

Los adolescentes dedican su tiempo diario a dos grandes bloques de actividades: a las actividades formales que engloban asistir a clase y realización de tareas escolares; y a las actividades de ocio y tiempo libre. En los últimos años, la variedad de posibilidades que se encuentra un adolescente para hacer en su tiempo libre ha aumentado considerablemente, desde el desarrollo de las nuevas tecnologías y el rápido y accesible acceso que tenemos a ellas, hasta la creación de nuevos espacios lúdicos como centros deportivos, culturales, asociaciones.... Todo ello, está incidiendo en nuevos y diferentes estilos de vida y también de aprendizaje y de estudio.

Las nuevas tecnologías son utilizadas cada vez de manera más frecuente por los adolescentes. Los dispositivos y herramientas que facilitan este uso de estas TIC están muy generalizados entre ellos. El acceso a través de un clic a cualquier tipo de información que necesiten, al ocio, a las relaciones sociales, a los servicios, etc...es algo que fascina a los adolescentes. Pero esta facilidad no está exenta de riesgos y saber identificarlos no es tarea sencilla para un adolescente que se encuentra en pleno proceso de su desarrollo madurativo

Por otra parte, las expectativas sobre la trascendencia de las innovaciones tecnológicas son cada vez mayores, desde diversos puntos de vista. $Y$ de manera especial hay que considerar su capacidad de transformación social global (Attali, 2006). Hoy día, la expectativa de una nueva generación competente en el uso de las TIC parece tener una situación de ventaja y cierto dominio sobre el resto de la población que no haya adquirido estas competencias (Rodríguez San Julián, Ballesteros Guerra, Megías Quirós (2011), y García Fernández (2009)).

Esta nueva realidad, está produciendo cambios en los estilos de vida y de aprendizaje de nuestros jóvenes, por ello, queremos presentar algunos datos en relación con la repetición de cursos escolares y el uso inadecuado de las TIC. 


\section{METODOLOGÍA}

\section{Objetivo}

Conocer los hábitos en relación con el uso y/o abuso de las TIC, en una muestra de adolescentes escolarizados en la Comunidad Autónoma de Cantabria que han repetido Curso académico.

\section{Muestra}

Se ha realizado un estudio en la C.A. de Cantabria, con una muestra de 2371 alumnos, del rango de cursos desde $1^{\circ}$ de ESO hasta $1^{\circ}$ de Bachillerato y presenta una distribución de 1168 mujeres y1203 varones, con un muestreo por estratos y conglomerados para que la muestra pudiera ser considerada representativa de la Comunidad Autónoma, y una de las cuestiones analizadas es la de las repeticiones de curso y su posible relación con el uso y abuso de las TIC.

\section{Instrumentos}

Para la recogida de datos se utilizó el método de encuesta, realizada de forma presencial en cada centro educativo, aunque administrada de forma online en cuanto a la presentación y recogida de los datos. Este tipo de encuesta, se considera un instrumento adecuado para acceder a la conducta, conocimiento y procesos educativos. La encuesta usada en esta investigación se califica como:

\section{Muestral}

Se ha encuestado a una muestra representativa de alumnos de la ESO y de 1 de Bachillerato de toda la Comunidad Autónoma de Cantabria (previamente zonificada), contemplando tanto a la enseñanza pública como de la concertada.

Descriptiva y explicativa Se aportan los resultados en términos de estadísticos descriptivos y se pone de manifiesto la relación entre las variables objeto de estudio.

Transversal Se ha recogido la información en un momento concreto, en una única sesión.

Para la elaboración del instrumento se utilizaron las revisiones bibliográficas, la opinión de expertos externos, así como la opinión de alumnos pertenecientes a la población diana. Una vez confeccionado el cuestionario, se realizó una prueba piloto que se aplicó a una pequeña muestra de sujetos homogéneos a la población estudiada. Esta aplicación permitió realizar una nueva revisión que es fue la definitiva.

\section{Procedimiento}

Con el fin de que la administración de la prueba se produjera en condiciones similares para todos los sujetos y así evitar los sesgos que se pudieran producir por parte del encuestador, la responsable del trabajo asistió a las sesiones para la obtención de los datos. En los centros a los que no pudo asistir se instruyó a los profesores para que el procedimiento fuera igual en todas las situaciones.

La presentación online de la encuesta permitió evitar posibles situaciones anómalas en la aplicación e incorporar las respuestas directamente a una base de datos, por ello se optó por este método en la práctica totalidad de los casos.

\section{RESULTADOS}

Una constatación inicial general, es el mucho tiempo que una buena parte de los adolescentes de la muestra dedican a interactuar con los diferentes dispositivos tecnológicos. En especial todo lo relacionado con la informática, Internet, redes sociales, y teléfonos inteligentes, WhatsApp, parece que cada vez es utilizado de manera más frecuente, y tiene un gran atractivo para los adolescentes, 


\section{USO Y ABUSO DE LAS TIC Y REPETICIÓN DE CURSO EN ADOLESCENTES}

pero sin olvidar algunos otros medios como la televisión que hoy todavía tiene una gran presencia en nuestras vidas.

A continuación, se irán presentando algunos de los resultados analizados al respecto y su posible relación con las repeticiones de cursos escolares.

\section{Repite curso}

En la distribución de alumnos que han repetido curso se observa que un $77,22 \%$ nunca han repetido curso, por lo que un $22,77 \%$, que es un porcentaje nada desdeñable, sí que ha repetido. De ellos, un $18,22 \%$ ha repetido un único curso, y un $4,55 \%$ han repetido dos o más cursos.

Tabla 1. Repite curso

\begin{tabular}{|l|c|c|}
\hline \multicolumn{1}{|c|}{ Repite curso } & Frecuencia & Porcentaje \\
\hline - No & 1831 & $77,22 \%$ \\
\hline 1 curso & 432 & $18,22 \%$ \\
\hline - 2 cursos & 79 & $3,33 \%$ \\
\hline
\end{tabular}

El porcentaje de alumnos que han repetido dos cursos en la ESO es del 3,33\% por lo que no podrán repetir ningún curso más en ese nivel educativo. En bachillerato este porcentaje es del $1,22 \%$. En primer curso de la ESO han repetido una vez un $18.22 \%$ de los alumnos. Un total del $22,77 \%$ de los alumnos de la muestra ha repetido curso, lo que sin duda requiere una reflexión, pues $\mathrm{s}$ e trata de un número importante de alumnos.

\section{Calificación media del último curso}

Tabla 2. Calificación media último curso

\begin{tabular}{|c|c|c|}
\hline & Varón & Mujer \\
\hline - Insuficiente & $8,31 \%$ & $6,08 \%$ \\
\hline - Suficiente & $18,20 \%$ & $15,58 \%$ \\
\hline - Bien & $28,76 \%$ & $25,94 \%$ \\
\hline - Notable & $35,74 \%$ & $38,36 \%$ \\
\hline - Sobresaliente & $8,98 \%$ & $14,04 \%$ \\
\hline
\end{tabular}

En cuanto a las calificaciones medias obtenidas en el último curso, se aprecia una distribución con un peso mayor en las calificaciones más altas de notable y sobresaliente, que las de insuficien- 
te y suficiente (Tabla 2). Los porcentajes más elevados en cuanto a las calificaciones se corresponden con las categorías de Bien con un 25,94\% y de Notable con un 38,36\%. Las diferencias en las calificaciones obtenidas en el curso anterior parecen ser ligeramente más favorables a las mujeres. El porcentaje de insuficientes es del $8,31 \%$ en varones y del $6,08 \%$ en mujeres.

\section{Relación de repite curso con diferentes TIC:}

En este apartado, se van a considerar los cruces de contrastes que han resultado estadísticamente significativos, relacionando la repetición de cursos escolares, con algunas variables relacionadas con la percepción que tienen los alumnos sobre tecnologías consideradas relevantes para nuestra investigación.

\section{Televisor}

Tabla 3. Repite curso*televisor

\begin{tabular}{|c|c|c|c|c|c|c|c|c|c|}
\hline & & & \multicolumn{6}{|c|}{ Televisor } & \multirow[b]{2}{*}{ Total } \\
\hline & & & No lo tengo & \begin{tabular}{|c}
$\begin{array}{c}\text { Imprescin } \\
\text { dible }\end{array}$ \\
\end{tabular} & $\begin{array}{c}\text { Interesante, } \\
\text { divertido }\end{array}$ & $\begin{array}{l}\text { Superfluo, } \\
\text { indiferente }\end{array}$ & Aburrido, inútil & Molesto & \\
\hline \multirow{6}{*}{$\begin{array}{l}\text { Repite } \\
\text { curso }\end{array}$} & \multirow[t]{2}{*}{ No } & Recuento & 7 & 459 & 961 & 327 & 68 & 9 & 1831 \\
\hline & & $\%$ de Repite curso & $.4 \%$ & $25,1 \%$ & $52,5 \%$ & $17,9 \%$ & $3,7 \%$ &, $5 \%$ & $100,0 \%$ \\
\hline & \multirow[t]{2}{*}{$\mathrm{Si}$, un curso } & Recuento & 2 & 133 & 190 & 77 & 27 & 3 & 432 \\
\hline & & $\%$ de Repite curso &, $5 \%$ & $30,8 \%$ & $44,0 \%$ & $17,8 \%$ & $6,3 \%$ &, $7 \%$ & $100,0 \%$ \\
\hline & \multirow[t]{2}{*}{ Si, dos cursos } & Recuento & 1 & 35 & 53 & 17 & 2 & 0 & 108 \\
\hline & & $\%$ de Repite curso &, $9 \%$ & $32,4 \%$ & $49,1 \%$ & $15,7 \%$ & $1,9 \%$ &, $0 \%$ & $100,0 \%$ \\
\hline \multirow[t]{2}{*}{ Total } & & Recuento & 10 & 627 & 1204 & 421 & 97 & 12 & 2371 \\
\hline & & $\%$ de Repite curso & $.4 \%$ & $26,4 \%$ & $50,8 \%$ & $17,8 \%$ & $4,1 \%$ & $5 \%$ & $100,0 \%$ \\
\hline
\end{tabular}

Tabla 4. Pruebas de chi-cuadrado repite curso*televisor

Pruebas de chi-cuadrado

\begin{tabular}{|l|c|r|r|}
\hline & \multicolumn{1}{|c|}{ Valor } & \multicolumn{1}{|c|}{ gl } & \multicolumn{1}{c|}{$\begin{array}{c}\text { Sig. asintótica } \\
\text { (bilateral) }\end{array}$} \\
\hline Chi-cuadrado de Pearson & $19,621^{\mathrm{g}}$ & 10 &, 033 \\
Razón de verosimilitudes & 19,598 & 10 &, 033 \\
Asociación lineal por & 1,973 & 1 &, 160 \\
lineal & 2371 & & \\
N de casos válidos & & \\
\hline
\end{tabular}

a. 5 casillas $(27,8 \%)$ tienen una frecuencia esperada inferior a 5 . La frecuencia mínima esperada es, 46 .

Se aprecia una diferencia significativa entre los alumnos que han repetido algún curso y los que no en la percepción que pueden tener de la televisión, como algo imprescindible (Tabla 4). A medida que se repiten cursos, más imprescindible manifiestan que les resulta la televisión. Los alumnos que han repetido dos cursos en un porcentaje de un $81,5 \%$ consideran imprescindible 0 interesante y divertida la televisión, los que han repetido un solo curso lo consideran en un $74,8 \%$. Si analizamos únicamente la opción de quienes valoran a este medio imprescindible, vemos que los porcentajes son del 21,5\% para quienes no han repetido curso, del 30,8 \% para quienes han repetido un único curso y del $32,4 \%$ para los que han repetido dos o más curos. Tan solo un $17 \%$ lo consideran superfluo, siendo muy residuales el resto de los porcentajes (Tabla 3). 


\section{USO Y ABUSO DE LAS TIC Y REPETICIÓN DE CURSO EN ADOLESCENTES}

\section{Ordenador}

Tabla 2

Repite curso*ordenador

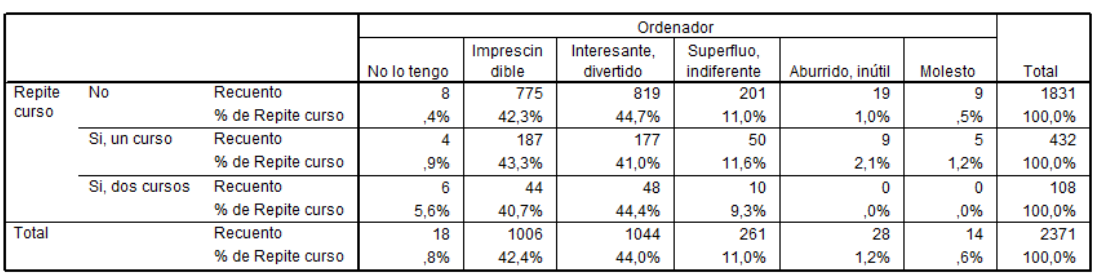

Tabla 3

Pruebas de chi-cuadrado repite curso*ordenador

Pruebas de chi-cuadrado

\begin{tabular}{|l|c|r|r|}
\hline & Valor & gl & \multicolumn{1}{|c|}{$\begin{array}{c}\text { Sig. asintótica } \\
\text { (bilateral) }\end{array}$} \\
\hline Chi-cuadrado de Pearson & $44,960^{\circledR}$ & 10 &, 000 \\
Razón de verosimilitudes & 27,107 & 10 &, 003 \\
Asociación lineal por &, 751 & 1 &, 386 \\
lineal & 2371 & & \\
N de casos válidos & & & \\
\hline
\end{tabular}

a. 5 casillas $(27,8 \%)$ tienen una frecuencia esperada inferior a 5 . La frecuencia mínima esperada es , 64 .

La percepción que tienen de, lo imprescindible, interesante, superfluo, aburrido, o molesto que les resulte el ordenador, no parece que tenga una relación con la repetición de cursos escolares. Lo que, si se aprecian diferencias estadísticamente significativas en relación con que una muy amplia mayoría de los encuestados, consideran al ordenador, como algo imprescindible, interesante 0 divertido (Tabla 6). Un 0,8\% no tiene ordenador en el hogar (suele darse esta infrecuente situación en las zonas más rurales). El 86,4\% lo consideran imprescindible 0 interesante. Siendo considerado superfluo 0 indiferente por un $11 \%$ de los encuestados e inútil, aburrido 0 molesto por un porcentaje residual del 1,8\% (Tabla 5).

\section{Internet}

Tabla 4

Repite curso * Internet

\begin{tabular}{|c|c|c|c|c|c|c|c|c|c|}
\hline & & & \multicolumn{6}{|c|}{ Internet } & \multirow[b]{2}{*}{ Total } \\
\hline & & & No lo tengo & $\begin{array}{c}\text { Imprescin } \\
\text { dible }\end{array}$ & $\begin{array}{c}\text { Interesante, } \\
\text { divertido }\end{array}$ & $\begin{array}{l}\text { Superfluo, } \\
\text { indiferente }\end{array}$ & Aburrido, inútil & Molesto & \\
\hline \multirow{6}{*}{$\begin{array}{l}\text { Repite } \\
\text { curso }\end{array}$} & \multirow[t]{2}{*}{ No } & Recuento & 12 & 1311 & 427 & 68 & 7 & 6 & 1831 \\
\hline & & $\%$ de Repite curso &, $7 \%$ & $71,6 \%$ & $23,3 \%$ & $3,7 \%$ & $.4 \%$ &, $3 \%$ & $100,0 \%$ \\
\hline & \multirow[t]{2}{*}{$\mathrm{Si}$, un curso } & Recuento & 7 & 299 & 102 & 18 & 5 & 1 & 432 \\
\hline & & $\%$ de Repite curso & $1,6 \%$ & $69,2 \%$ & $23,6 \%$ & $4,2 \%$ & $1,2 \%$ &, $2 \%$ & $100,0 \%$ \\
\hline & \multirow[t]{2}{*}{ Si, dos cursos } & Recuento & 5 & 71 & 28 & 3 & 0 & 1 & 108 \\
\hline & & $\%$ de Repite curso & $4,6 \%$ & $65,7 \%$ & $25,9 \%$ & $2,8 \%$ &, $0 \%$ &, $9 \%$ & $100,0 \%$ \\
\hline \multirow[t]{2}{*}{ Total } & & Recuento & 24 & 1681 & 557 & 89 & 12 & 8 & 2371 \\
\hline & & $\%$ de Repite curso & $1,0 \%$ & $70,9 \%$ & $23,5 \%$ & $3,8 \%$ &, $5 \%$ &, $3 \%$ & $100,0 \%$ \\
\hline
\end{tabular}


Tabla 8.

Pruebas de chi-cuadrado repite curso * Internet

Pruebas de chi-cuadrado

\begin{tabular}{|l|c|r|r|}
\hline & Valor & gl & $\begin{array}{c}\text { Sig. asintótica } \\
\text { (bilateral) }\end{array}$ \\
\hline Chi-cuadrado de Pearson & $29,019^{\mathrm{a}}$ & 10 &, 001 \\
Razón de verosimilitudes & 31,269 & 10 &, 001 \\
Asociación lineal por & 2,364 & 1 &, 124 \\
lineal & 2371 & & \\
N de casos válidos & & & \\
\hline
\end{tabular}

a. 6 casillas $(33,3 \%)$ tienen una frecuencia esperada inferior a 5 . La frecuencia mínima esperada es 2,47 .

De nuevo vemos como Internet tiene un enorme atractivo para los adolescentes, el 70,9\% tanto de repetidores como de no repetidores, consideran que la red es para ellos imprescindible, y un porcentaje entre el $23,5 \%$ lo consideran como interesante, o divertido. Lo que hace que un porcentaje próximo al 95\% manifiestan claramente la importancia que tiene para ellos la red, lo que resulta estadísticamente significativo (Tabla 8). Si analizamos más en detalle los resultados obtenidos, se aprecia que se considera Internet imprescindible por al $71,6 \%$ de los alumnos no repetidores, bajando el porcentaje de esta percepción a medida que repiten curso (Tabla 7).

\section{Videoconsola}

Tabla 9 .

Repite curso * videoconsola

\begin{tabular}{|c|c|c|c|c|c|c|c|c|c|}
\hline & & & \multicolumn{6}{|c|}{ Videoconsola } & \multirow[b]{2}{*}{ Total } \\
\hline & & & No lo tengo & $\begin{array}{c}\text { Imprescin } \\
\text { dible }\end{array}$ & $\begin{array}{c}\text { Interesante, } \\
\text { divertido }\end{array}$ & $\begin{array}{l}\text { Superfluo, } \\
\text { indiferente }\end{array}$ & Aburrido, inútil & Molesto & \\
\hline \multirow{6}{*}{$\begin{array}{l}\text { Repite } \\
\text { curso }\end{array}$} & No & Recuento & 121 & 326 & 596 & 513 & 211 & 64 & 1831 \\
\hline & & $\%$ de Repite curso & $6,6 \%$ & $17,8 \%$ & $32,6 \%$ & $28,0 \%$ & $11,5 \%$ & $3,5 \%$ & $100,0 \%$ \\
\hline & $\mathrm{Si}$, un curso & Recuento & 56 & 78 & 125 & 106 & 49 & 18 & 432 \\
\hline & & $\%$ de Repite curso & $13,0 \%$ & $18,1 \%$ & $28,9 \%$ & $24,5 \%$ & $11,3 \%$ & $4,2 \%$ & $100,0 \%$ \\
\hline & Si, dos cursos & Recuento & 12 & 20 & 25 & 38 & 11 & 2 & 108 \\
\hline & & $\%$ de Repite curso & $11,1 \%$ & $18,5 \%$ & $23,1 \%$ & $35,2 \%$ & $10,2 \%$ & $1,9 \%$ & $100,0 \%$ \\
\hline \multirow[t]{2}{*}{ Total } & & Recuento & 189 & 424 & 746 & 657 & 271 & 84 & 2371 \\
\hline & & $\%$ de Repite curso & $8,0 \%$ & $17,9 \%$ & $31,5 \%$ & $27,7 \%$ & $11,4 \%$ & $3,5 \%$ & $100,0 \%$ \\
\hline
\end{tabular}

Tabla 5

Pruebas de chi-cuadrado repite curso * videoconsola

Pruebas de chi-cuadrado

\begin{tabular}{|l|r|r|r|}
\hline & Valor & gl & \multicolumn{1}{|c|}{$\begin{array}{c}\text { Sig. asintótica } \\
\text { (bilateral) }\end{array}$} \\
\hline Chi-cuadrado de Pearson & $28,413^{\text {a }}$ & 10 &, 002 \\
Razón de verosimilitudes & 26,676 & 10 &, 003 \\
Asociación lineal por & 4,214 & 1 &, 040 \\
lineal & 2371 & & \\
N de casos válidos & & & \\
\hline
\end{tabular}

a. 1 casillas $(5,6 \%)$ tienen una frecuencia esperada inferior a 5 . La frecuencia mínima esperada es 3,83 . 


\section{USO Y ABUSO DE LAS TIC Y REPETICIÓN DE CURSO EN ADOLESCENTES}

La videoconsola, parece tener menos interés para los componentes de la muestra. Y tampoco parece tener una relación que explique la repetición de cursos escolares. Lo que sí aparecen diferencias estadísticamente significativas entre los diferentes grupos de alumnos (Tabla 10). No tienen este dispositivo un $8,0 \%$ de los encuestados, pero son los alumnos repetidores los que en porcentajes mayores nos indican no disponer de videoconsola, con un $13 \%$ de quienes han repetido un solo curso y del $11,1 \%$ de quienes han repetido dos o más cursos (Tabla 9). El 42,6\% de la muestra considera a este dispositivo como algo superfluo, aburrido, podo útil e incluso molesto.

\section{Enganchado a Internet}

Tabla 6

Repite curso * enganchado a Internet

\begin{tabular}{|c|c|c|c|c|c|c|c|c|}
\hline & & & \multicolumn{5}{|c|}{ Enganchado a internet } & \multirow[b]{2}{*}{ Total } \\
\hline & & & Nada & Apenas & $\begin{array}{c}\text { Mediana } \\
\text { mente }\end{array}$ & Bastante & Mucho & \\
\hline \multirow{6}{*}{$\begin{array}{l}\text { Repite } \\
\text { curso }\end{array}$} & \multirow[t]{2}{*}{ No } & Recuento & 124 & 283 & 575 & 498 & 351 & 1831 \\
\hline & & $\%$ de Repite curso & $6,8 \%$ & $15,5 \%$ & $31,4 \%$ & $27,2 \%$ & $19,2 \%$ & $100,0 \%$ \\
\hline & \multirow[t]{2}{*}{$\mathrm{Si}$, un curso } & Recuento & 32 & 65 & 127 & 108 & 100 & 432 \\
\hline & & $\%$ de Repite curso & $7,4 \%$ & $15,0 \%$ & $29,4 \%$ & $25,0 \%$ & $23,1 \%$ & $100,0 \%$ \\
\hline & \multirow[t]{2}{*}{ Si, dos cursos } & Recuento & 9 & 18 & 26 & 20 & 35 & 108 \\
\hline & & $\%$ de Repite curso & $8,3 \%$ & $16,7 \%$ & $24,1 \%$ & $18,5 \%$ & $32,4 \%$ & $100,0 \%$ \\
\hline \multirow[t]{2}{*}{ Total } & & Recuento & 165 & 366 & 728 & 626 & 486 & 2371 \\
\hline & & $\%$ de Repite curso & $7,0 \%$ & $15,4 \%$ & $30,7 \%$ & $26,4 \%$ & $20,5 \%$ & $100,0 \%$ \\
\hline
\end{tabular}

Tabla 12

Pruebas de chi-cuadrado repite curso * enganchado a Internet

Pruebas de chi-cuadrado

\begin{tabular}{|c|c|c|c|}
\hline & Valor & gl & $\begin{array}{l}\text { Sig. asintótica } \\
\text { (bilateral) }\end{array}$ \\
\hline Chi-cuadrado de Pearson & $16,672^{9}$ & 8 &, 034 \\
\hline Razón de verosimilitudes & 16,382 & 8 &, 037 \\
\hline $\begin{array}{l}\text { Asociación lineal por } \\
\text { lineal }\end{array}$ &, 002 & 1 & ,968 \\
\hline $\mathrm{N}$ de casos válidos & 2371 & & \\
\hline
\end{tabular}

a. 0 casillas $(, 0 \%)$ tienen una frecuencia esperada inferior a 5 . La frecuencia mínima esperada es 50,87 .

El 32,4\% de los alumnos que han repetido dos cursos nos responden que están muy enganchados a Internet. Y un $23,1 \%$ de los que han repetido un curso también nos indican que lo están, frente al $19,2 \%$ de quienes no han repetido ningún curso. Solamente un $7 \%$ de los encuestados, nos manifiestan no estar nada enganchados a Internet. Un 46,9\% nos señalan que se consideran bastante 0 muy enganchados a la red (Tabla 11). El considerarse enganchado a Internet, sí que parece que pudiera tener una cierta relación con el rendimiento académico (Tabla 12). Habría que conocer también el uso que hace de ese tiempo que está conectado a la red. 


\section{Enganchado a redes sociales}

Tabla 13.

Repite curso * enganchado a redes sociales

\begin{tabular}{|c|c|c|c|c|c|c|c|c|}
\hline & & & \multicolumn{5}{|c|}{ Enganchado a redes sociales } & \multirow[b]{2}{*}{ Total } \\
\hline & & & Nada & Apenas & $\begin{array}{c}\text { Mediana } \\
\text { mente }\end{array}$ & Bastante & Mucho & \\
\hline \multirow{6}{*}{$\begin{array}{l}\text { Repite } \\
\text { curso }\end{array}$} & \multirow{2}{*}{ No } & Recuento & 230 & 332 & 482 & 452 & 335 & 1831 \\
\hline & & $\%$ de Repite curso & $12,6 \%$ & $18,1 \%$ & $26,3 \%$ & $24,7 \%$ & $18,3 \%$ & $100,0 \%$ \\
\hline & \multirow[t]{2}{*}{$\mathrm{Si}$, un curso } & Recuento & 45 & 63 & 111 & 102 & 111 & 432 \\
\hline & & $\%$ de Repite curso & $10,4 \%$ & $14,6 \%$ & $25,7 \%$ & $23,6 \%$ & $25,7 \%$ & $100,0 \%$ \\
\hline & \multirow[t]{2}{*}{ Si, dos cursos } & Recuento & 15 & 12 & 24 & 29 & 28 & 108 \\
\hline & & $\%$ de Repite curso & $13,9 \%$ & $11,1 \%$ & $22,2 \%$ & $26,9 \%$ & $25,9 \%$ & $100,0 \%$ \\
\hline \multirow{2}{*}{\multicolumn{2}{|c|}{ Total }} & Recuento & 290 & 407 & 617 & 583 & 474 & 2371 \\
\hline & & $\%$ de Repite curso & $12,2 \%$ & $17,2 \%$ & $26,0 \%$ & $24,6 \%$ & $20,0 \%$ & $100,0 \%$ \\
\hline
\end{tabular}

Tabla 7

Pruebas de chi-cuadrado repite curso * enganchado a redes sociales

Pruebas de chi-cuadrado

\begin{tabular}{|c|c|c|c|}
\hline & Valor & $\mathrm{gl}$ & $\begin{array}{l}\text { Sig. asintótica } \\
\text { (bilateral) }\end{array}$ \\
\hline Chi-cuadrado de Pearson & $19,195^{a}$ & 8 & ,014 \\
\hline Razón de verosimilitudes & 19,004 & 8 &, 015 \\
\hline $\begin{array}{l}\text { Asociación lineal por } \\
\text { lineal }\end{array}$ & 10,325 & 1 & 001 \\
\hline $\mathrm{N}$ de casos válidos & 2371 & & \\
\hline
\end{tabular}

a. 0 casillas $(, 0 \%)$ tienen una frecuencia esperada inferior a 5 . La frecuencia mínima esperada es 13,21.

Los porcentajes de alumnos que se manifiestan como enganchados medianamente, bastante 0 mucho, a las redes sociales representa a una amplia mayoría con porcentajes entre prácticamente el $70 \%$. Sí que parece que los alumnos repetidores declaran estar muy enganchado a estas redes en un porcentaje mayor, prácticamente un $26 \%$ de que quienes han repetido curso consideran que están muy enganchados (tanto de quienes ha repetido un curso, como de quienes han repetido dos; frente al 18,3\% de elección de esta opción por aquellos que no han repetido ningún curso (Tabla 13). Esta variable, sí que parece que pudiera tener una relación estadísticamente significativa con las repeticiones de curso (Tabla 14).

\section{Enganchado a teléfono móvil}

Tabla 8

Repite curso * enganchado a teléfono móvil

\begin{tabular}{|c|c|c|c|c|c|c|c|c|}
\hline & & & \multicolumn{5}{|c|}{ Enganchado a teléfono móvil } & \multirow[b]{2}{*}{ Total } \\
\hline & & & Nada & Apenas & $\begin{array}{c}\text { Mediana } \\
\text { mente }\end{array}$ & Bastante & Mucho & \\
\hline \multirow{6}{*}{$\begin{array}{l}\text { Repite } \\
\text { curso }\end{array}$} & \multirow{2}{*}{ No } & Recuento & 248 & 247 & 434 & 469 & 433 & 1831 \\
\hline & & $\%$ de Repite curso & $13,5 \%$ & $13,5 \%$ & $23,7 \%$ & $25,6 \%$ & $23,6 \%$ & $100,0 \%$ \\
\hline & \multirow[t]{2}{*}{ Si, un curso } & Recuento & 42 & 59 & 101 & 98 & 132 & 432 \\
\hline & & $\%$ de Repite curso & $9,7 \%$ & $13,7 \%$ & $23,4 \%$ & $22,7 \%$ & $30,6 \%$ & $100,0 \%$ \\
\hline & \multirow[t]{2}{*}{ Si, dos cursos } & Recuento & 10 & 16 & 18 & 26 & 38 & 108 \\
\hline & & $\%$ de Repite curso & $9,3 \%$ & $14,8 \%$ & $16,7 \%$ & $24,1 \%$ & $35,2 \%$ & $100,0 \%$ \\
\hline \multirow{2}{*}{\multicolumn{2}{|c|}{ Total }} & Recuento & 300 & 322 & 553 & 593 & 603 & 2371 \\
\hline & & $\%$ de Repite curso & $12,7 \%$ & $13,6 \%$ & $23,3 \%$ & $25,0 \%$ & $25,4 \%$ & $100,0 \%$ \\
\hline
\end{tabular}




\section{USO Y ABUSO DE LAS TIC Y REPETICIÓN DE CURSO EN ADOLESCENTES}

Tabla 9

Pruebas de chi-cuadrado repite curso * enganchado a teléfono móvil

Pruebas de chi-cuadrado

\begin{tabular}{|c|c|c|c|}
\hline & Valor & gl & $\begin{array}{l}\text { Sig. asintótica } \\
\text { (bilateral) }\end{array}$ \\
\hline Chi-cuadrado de Pearson & $19,394^{9}$ & 8 & ,013 \\
\hline Razón de verosimilitudes & 19,328 & 8 &, 013 \\
\hline $\begin{array}{l}\text { Asociación lineal por } \\
\text { lineal }\end{array}$ & 10,173 & 1 &, 001 \\
\hline $\mathrm{N}$ de casos válidos & 2371 & & \\
\hline
\end{tabular}

a. 0 casillas $(, 0 \%)$ tienen una frecuencia esperada inferior a 5 . La frecuencia mínima esperada es 13,67.

Los alumnos repetidores sí que manifiestan que están enganchados al móvil en porcentajes mayores que aquellos otros que no han repetido curso. Este hecho, sí que puede implicar una relación estadísticamente significativa (Tabla 16) con las repeticiones de curso. En todo caso, unos porcentajes importantes de entre el $53,3 \%$ y el $59,3 \%$ de quienes han repetido uno 0 dos cursos, sí que se declaran enganchados al teléfono móvil (Tabla 15).

\section{Enganchado a Whatsapp}

Tabla 10

Repite curso * enganchado a WhatsApp

\begin{tabular}{|c|c|c|c|c|c|c|c|c|}
\hline & \multicolumn{5}{|c|}{ Enganchado a whatsapp } & \multirow[b]{2}{*}{ Total } \\
\hline & & & Nada & Apenas & $\begin{array}{l}\text { Mediana } \\
\text { mente }\end{array}$ & Bastante & Mucho & \\
\hline \multirow{6}{*}{$\begin{array}{l}\text { Repite } \\
\text { curso }\end{array}$} & \multirow[t]{2}{*}{ No } & Recuento & 343 & 153 & 312 & 452 & 571 & 1831 \\
\hline & & $\%$ de Repite curso & $18,7 \%$ & $8,4 \%$ & $17,0 \%$ & $24,7 \%$ & $31,2 \%$ & $100,0 \%$ \\
\hline & \multirow[t]{2}{*}{ Si, un curso } & Recuento & 85 & 30 & 72 & 77 & 168 & 432 \\
\hline & & $\%$ de Repite curso & $19,7 \%$ & $6,9 \%$ & $16,7 \%$ & $17,8 \%$ & $38,9 \%$ & $100,0 \%$ \\
\hline & \multirow[t]{2}{*}{ Si, dos cursos } & Recuento & 12 & 12 & 13 & 26 & 45 & 108 \\
\hline & & $\%$ de Repite curso & $11,1 \%$ & $11,1 \%$ & $12,0 \%$ & $24,1 \%$ & $41,7 \%$ & $100,0 \%$ \\
\hline \multirow[t]{2}{*}{ Total } & & Recuento & 440 & 195 & 397 & 555 & 784 & 2371 \\
\hline & & $\%$ de Repite curso & $18,6 \%$ & $8,2 \%$ & $16,7 \%$ & $23,4 \%$ & $33,1 \%$ & $100,0 \%$ \\
\hline
\end{tabular}

Tabla 11

Pruebas de chi cuadrado repite curso * enganchado a WhatsApp

Pruebas de chi-cuadrado

\begin{tabular}{|c|c|c|c|}
\hline & Valor & $\mathrm{gl}$ & $\begin{array}{l}\text { Sig. asintótica } \\
\text { (bilateral) }\end{array}$ \\
\hline Chi-cuadrado de Pearson & $22,925^{9}$ & 8 &, 003 \\
\hline Razón de verosimilitudes & 23,673 & 8 &, 003 \\
\hline $\begin{array}{l}\text { Asociación lineal por } \\
\text { lineal }\end{array}$ & 4,990 & 1 &, 025 \\
\hline $\mathrm{N}$ de casos válidos & 2371 & & \\
\hline
\end{tabular}

a. 0 casillas $(, 0 \%)$ tienen una frecuencia esperada inferior a 5 . La frecuencia mínima esperada es 8,88 .

En relación con los que dicen estar enganchados al WhatsApp y la repetición de curso, sí que se encuentran claras diferencias significativas estadísticamente (Tabla 18). Aquellos alumnos que 
han repetido dos cursos en un 41,7\% declaran estar muy enganchados a esta aplicación, y el 24,4\% se consideran bastante enganchados, lo que supone prácticamente un $66 \%$. Entre quienes han repetido un curso el porcentaje baja a cerca del $57 \%$ (Tabla 17), en cualquier caso, si que se encuentran diferencias estadísticamente significativas entre los grupos (Tabla 18).

En cualquier caso, esta aplicación tiene un creciente atractivo para los adolescentes que en porcentajes superiores al $72,9 \%$ hacen bastante uso de esta.

\section{CONCLUSIONES}

Un $22,77 \%$ de los alumnos de la muestra han repetido curso, lo que sin duda requiere una reflexión pues no deja de ser un porcentaje nada desdeñable. Los que sí han repetido algún curso usan más las nuevas tecnologías y de manera menos adecuada, en comparación con los que no han repetido curso.

Los alumnos que han repetido curso ven más televisión que los no repetidores. A medida que se repiten cursos, más imprescindible manifiestan que les resulta la televisión.

Internet tiene un enorme atractivo para todos los adolescentes. Si analizamos en detalle los resultados obtenidos, se aprecia que el porcentaje de alumnos que consideran imprescindible para ellos Internet baja a medida que repiten curso. El porcentaje de los alumnos que han repetido dos cursos y responden que están muy enganchados a Internet. $Y$ de los que han repetido un curso y también nos indican que lo están, es superior de quienes no han repetido ningún curso y eligen esta opción.

Los alumnos repetidores sí que manifiestan que están enganchados al móvil en porcentajes mayores que aquellos otros que no han repetido curso.

Aquellos alumnos que han repetido dos cursos en un porcentaje alto se declaran estar muy 0 bastante enganchados al WhatsApp. Entre quienes han repetido un curso, o ninguno, este porcentaje es mas bajo.

Los padres, y los educadores, debemos realizar un mayor esfuerzo educativo con respecto al consumo de los diferentes medios tecnológicos, incentivando una utilización racional y responsable de las TIC por parte de los adolescentes.

\section{REFERENCIAS BIBLIOGRÁFICAS}

Attali, J. (2006). Breve historia del futuro. Barcelona. Paidós Ibérica.

Barrio, A (2017). Las Tecnologías de la Información y la Comunicación en la vida y la educación de Ios adolescentes. UEX

García, F. (2009). Nativos interactivos: Ios adolescentes y sus pantallas: reflexiones educativas. Madrid. Foro Generaciones Interactiva.

Rodríguez San Julián, E., Ballesteros Guerra, JC., Megías Quirós, I. (2011). Bienestar en España. Ideas de Futuro desde el discurso de padres y madres. Madrid. FAD- Caja Madrid

Valdemoros, M.A., Sanz, E., y Ponce de León, A., (2017). Ocio digital y ambiente familiar en estudiantes de Educación Postobligatoria. Comunicar, 50 (XXV), 99-108 
